\title{
miR-130a-3p promotes cell proliferation and invasion by targeting estrogen receptor $\alpha$ and androgen receptor in cervical cancer
}

\author{
QIONG FAN ${ }^{1-4^{*}}$, TING HUANG ${ }^{1-4 *}$, XIAO SUN ${ }^{1-3}$, XIAOMING YANG $^{1}$, JING WANG $^{1}$, \\ YAO LIU ${ }^{1}$, TING NI ${ }^{1}$, SHENGLAN GU ${ }^{1}$, YUHONG LI ${ }^{1}$ and YUDONG WANG ${ }^{1-4}$ \\ ${ }^{1}$ Department of Gynecological Oncology, The International Peace Maternity and Child Health Hospital, \\ School of Medicine, Shanghai Jiao Tong University; ${ }^{2}$ Shanghai Key Clinical Department; \\ ${ }^{3}$ Shanghai Key Laboratory of Embryo Original Diseases Affiliated to Shanghai Jiao Tong University School of Medicine; \\ ${ }^{4}$ Shanghai Municipal Key Clinical Specialty, Shanghai 200030, P.R. China
}

Received January 30, 2020; Accepted August 11, 2020

DOI: $10.3892 / \mathrm{etm} .2021 .9858$

\begin{abstract}
Cervical cancer is the most common gynecological cancer in women worldwide. Human papillomavirus (HPV) is required but not sufficient for developing cervical cancer. HPV E6 and E7 proteins are able to directly interact with certain nuclear receptors; however, whether steroid hormone receptors mediate cervical carcinogenesis is not completely understood. The present study demonstrated via immunohistochemistry that estrogen receptor $\alpha(E R \alpha)$ and androgen receptor (AR) expression were decreased in a sequential manner from healthy cervical tissues to cervical intraepithelial neoplasia tissues and further to cervical cancer (CC) tissues, whereas microRNA (miR)-130a-3p expression levels were higher in $\mathrm{CC}$ tissues compared with healthy tissues. Both ER $\alpha$ and AR were direct targets of miR-130a-3p, as determined by performing luciferase reporter assays and western blotting. Functionally, compared with the corresponding control groups, miR-130a-3p knockdown, ER $\alpha$ overexpression and AR overexpression significantly inhibited $\mathrm{CC}$ cell proliferation and invasion, as demonstrated by the results obtained from the Cell Counting Kit-8 and Transwell assays in vitro. In addition, antagomiR-130a decreased tumor size and weight in vivo compared with control antagomiR as determined via the xenograft tumor growth assay. Therefore, the results suggested that miR-130a-3p might contribute to tumor progression by suppressing ER $\alpha$ and AR, and serve
\end{abstract}

Correspondence to: Professor Yudong Wang, Department of Gynecological Oncology, The International Peace Maternity and Child Health Hospital, School of Medicine, Shanghai Jiao Tong University, 910 Hengshan Road, Shanghai 200030, P.R. China

E-mail: wangyudong6688@126.com

*Contributed equally

Key words: cervical cancer, microRNA, estrogen receptor $\alpha$, androgen receptor, neoplasm, invasion as a promising candidate target for the treatment of patients with $\mathrm{CC}$.

\section{Introduction}

Cervical cancer (CC) is the most common gynecological cancer in women worldwide (1). In 2019, 13,170 new cases were diagnosed and 4,250 disease-related deaths were predicted in the USA (1). Furthermore, 530,000 new cases are diagnosed yearly worldwide and $20-25 \%$ of cases are reported in China (2). Although CC also occurs during perimenopause, unlike endometrial cancer or ovarian cancer, there are few reports on the association between steroid hormone receptors and CC (3). Several in vitro studies have investigated the interactions between estrogen/progesterone and human papillomavirus (HPV), but the results are controversial $(4,5)$.

Estrogen serves a major role in several hormone-dependent malignancies (6). Estrogen and its analogs can activate estrogen-responsive genes by binding to estrogen receptor $\alpha$ $(\mathrm{ER} \alpha)(7)$. Previous genomic studies have identified frequent mutations of the ER $\alpha$ gene (ESR1) in CC $(8,9)$. Additionally, it has been demonstrated that viral-codified E6 and E7 proteins could directly interact with androgen receptor (AR) (10). However, to the best of our knowledge, the role of ER $\alpha$ and AR in cervical carcinogenesis is not completely understood.

MircoRNAs (miRNAs/miRs) are a class of short, highly conserved, non-coding RNAs that regulate gene expression by inhibiting translation or inducing mRNA degradation at the post-transcriptional level (11). The aberrant expression of miRNAs has been reported in various tumors (12), such as endometrial cancer (13), ovarian cancer (14), and CC (15). Our previous study demonstrated that miR-107-5p could promote tumor proliferation and invasion by targeting ER $\alpha$ in endometrial carcinoma (16); therefore, it was hypothesized that specific miRNAs may control ER $\alpha$ and AR expression post-transcriptionally during CC progression.

The aim of the present study was to investigate the expression of miR-130a-3p, ER $\alpha$ and AR in healthy cervical and $\mathrm{CC}$ tissues, and to explore whether miR-130a-3p could contribute to tumor progression by suppressing ER $\alpha$ and AR. 


\section{Materials and methods}

Clinical samples. The present study was approved (approval no. GKLW 2017-125) by the Human Investigation Ethical Committee of the International Peace Maternity \& Child Hospital Affiliated to Shanghai Jiao Tong University School of Medicine (Shanghai, China). Written informed consent was obtained from all patients. A total of 60 female patients (45-68 years old) who underwent radical hysterectomy with lymph node dissection for $\mathrm{CC}$ at the International Peace Maternity \& Child Health Hospital Affiliated to Shanghai Jiao Tong University School of Medicine between August 2014 and April 2017 were included in the present study. The stages and histological grades of the tumors were determined according to the criteria of the Federation International of Gynecology and Obstetrics Surgical staging system (2018) (17). A total of 20 healthy cervical tissue samples (female subjects; 44-56 years old) were obtained from patients who underwent a hysterectomy to treat other diseases between April 2015 and April 2017 such as myoma or adenomyosis. In addition, 20 cervical intraepithelial neoplasia (CIN) I, 20 CIN II and 30 CIN III tissue samples (female subjects; 34-53 years old) were collected from patients who underwent a cervical biopsy or loop electrosurgical excision procedure between October 2015 and April 2017. All the tissues were collected from each patient or healthy control at the International Peace Maternity \& Child Health Hospital Affiliated to Shanghai Jiao Tong University School of Medicine and immediately snap-frozen in liquid nitrogen until further use. All the tissues were pathologically reviewed by two pathologists before use.

Immunohistochemistry (IHC). All tissue sections (4- $\mu$ m thick) were processed for IHC as previously described $(18,19)$. The following primary antibodies were used for IHC: Anti-ER $\alpha$ (cat. no. ab75635; 1:200; Abcam) and AR (cat. no. ab74272; 1:250; Abcam).

The expression of ER $\alpha$ and AR was evaluated in terms of staining intensity: (Negative), 1 (weak), 2 (medium) or 3 (strong). The extent of staining was scored as $0(0 \%), 1(1-25 \%)$, $2(26-50 \%), 3(51-75 \%)$ or $4(76-100 \%)$, according to the percentage of positively stained areas in relation to the whole tumor area. The final staining score $(0-12)$ was calculated by multiplying the intensity score and the extent score (19). The final staining score was used to evaluate the expression of ER $\alpha$ and AR.

Reverse transcription-quantitative PCR (RT-qPCR). Total RNA was extracted from frozen tissues using TRI Reagent (Molecular Research Center). Total RNA was reverse transcribed into mature miRNA using the TaqMan MicroRNA Reverse Transcription kit (Applied Biosystems; Thermo Fisher Scientific, Inc.), using the following RT temperature protocol: $42^{\circ} \mathrm{C}$ for $15 \mathrm{~min} ; 85^{\circ} \mathrm{C}$ for $5 \mathrm{sec}$. Subsequently, qPCR was performed using TaqMan MicroRNA assay primers (Applied Biosystems; Thermo Fisher Scientific, Inc.) with TaqMan Universal PCR Master mix (Applied Biosystems; Thermo Fisher Scientific, Inc.) and an ABI Prism 7000 Sequence Detection system (Applied Biosystems; Thermo Fisher Scientific, Inc.). The thermocycling conditions were as follows: $95^{\circ} \mathrm{C}$ for $3 \mathrm{~min} ; 95^{\circ} \mathrm{C}$ for $30 \mathrm{sec}$ and $60^{\circ} \mathrm{C}$ for $30 \mathrm{sec}$ for
35 cycles; $72^{\circ} \mathrm{C}$ for 5 min and maintenance at $4^{\circ} \mathrm{C}$ for further use. The sequences of the primers used for $\mathrm{qPCR}$ are provided in Table I. miRNA expression levels were normalized to the internal reference gene U6. Relative gene expression was quantified using the $2^{-\Delta \Delta \mathrm{Cq}}$ method (20).

Western blotting. Cells were harvested and proteins were extracted using Mem-PER Eukaryotic Membrane Protein Extraction Reagent (Pierce; Thermo Fisher Scientific, Inc.) containing complete mini cocktail, NE-PER Nuclear and Cytoplasmic Extraction Reagents (Pierce; Thermo Fisher Scientific, Inc.) and protease inhibitor cocktail. A total of $20 \mu \mathrm{g}$ proteins (determined using the BCA method) were separated via $8 \%$ SDS-PAGE and transferred onto PVDF membranes. The membranes were blocked for $1 \mathrm{~h}$ at room temperature with 5\% skimmed milk in TBS. Subsequently, the membranes were incubated with rabbit polyclonal primary antibodies targeted against: ER $\alpha$ (cat. no. ab75635; 1:1,000; Abcam), AR (cat. no. ab74272; 1:1,000; Abcam) and $\beta$-actin (cat. no. 4970; 1:2,000; Cell Signaling Technology, Inc.) in $10 \mathrm{ml}$ of $5 \%$ skimmed milk and incubated at $4^{\circ} \mathrm{C}$ overnight. After washing, the membranes were incubated with a horseradish peroxidase-conjugated goat anti-rabbit IgG secondary antibody (cat. no. 7074; 1:5,000; Cell Signaling Technology, Inc.) for $1 \mathrm{~h}$ at $37^{\circ} \mathrm{C}$. The results were visualized using an enhanced chemiluminescence kit (ECL kit; Pierce; Thermo Fisher Scientific, Inc.) using Kodak XAR-5 film (Sigma-Aldrich; Merck KGaA). $\beta$-actin was used as the loading control.

Cell culture and transfections. Human HeLa and SiHa CC cell lines, and 293T cells were obtained from The Cell Bank of Type Culture Collection of the Chinese Academy of Sciences. Cells were cultured in DMEM/F12 (cat. no. 11030; Gibco; Thermo Fisher Scientific, Inc.) supplemented with 10\% FBS (cat. no. 16000-44; Gibco; Thermo Fisher Scientific, Inc.) at $37^{\circ} \mathrm{C}$ with $5 \% \mathrm{CO}_{2}$.

miR-130a inhibitor (miR-130ai), miR-130a inhibitor negative control (NC; miR-130ai NC), miR-130a mimic (miR-130am) and miR-130a mimic negative control (miR-130am NC) were synthesized by Shanghai GenePharma Co., Ltd. The oligo sequences are provided in Table I. For transfection, cells were seeded into 6-well plates at 70-80\% confluence and grown overnight. Subsequently, HeLa or SiHa cells were transfected with $100 \mathrm{nM}$ miR-130ai, miR-130am, miR-130ai NC or miR-130am NC using Lipofectamine $^{\circledR} 2000$ (Invitrogen; Thermo Fisher Scientific, Inc.) according to the manufacturer's instructions. Transfection efficiency of miR-130ai and miR-130am was assessed via RT-qPCR after $24 \mathrm{~h}$ (Fig. S1A and B). For vector transduction, ER $\alpha$-overexpression vector (Ubi-MCS-3FLAG-ESR1), AR-overexpression vector (Ubi-MCS-3FLAG-AR) and empty vector were purchased from Shanghai GeneChem Co., Ltd. HeLa and SiHa cells were transfected with $1 \mu \mathrm{g} / \mathrm{ml}$ Ubi-MCS-3FLAG-ESR1, Ubi-MCS-3FLAG-AR or empty vector using Lipofectamine ${ }^{\circledR} 2000$ according to the manufacturer's instructions. Transfection efficiency of Ubi-MCS-3FLAG-ESR1 and Ubi-MCS-3FLAG-AR were confirmed via western blotting after $48 \mathrm{~h}$ (Fig. S1C and D).

Cell Counting Kit-8 (CCK-8) and cell clonogenic assays. To assess cell proliferation using the CCK-8 assay at $70-80 \%$ 
Table I. Oligo sequences used in the present study.

\begin{tabular}{|c|c|}
\hline Identifier & Sequence $\left(5^{\prime} \rightarrow 3^{\prime}\right)$ \\
\hline \multirow[t]{2}{*}{ miR-130a } & F: TTGCGATTCTGTTTTGTGCT \\
\hline & R: GTGGGGTCCTCAGTGGG \\
\hline \multirow[t]{2}{*}{ U6 } & F: AGAGCCTGTGGTGTCCG \\
\hline & R: CATCTTCAAAGCACTTCCCT \\
\hline \multirow[t]{2}{*}{ miR-130a inhibitor } & F: AUGCCCUUUUAACAUUGCACUG \\
\hline & R: UAGUGCAAUAGUAUCGUCGAGAC \\
\hline \multirow[t]{2}{*}{ miR-130a inhibitor NC } & F: CAGUACUUUUGUGUAGUACAA \\
\hline & R: GUCCUGAGAAGGCUAGCAUAGAU \\
\hline \multirow[t]{2}{*}{ miR-130a mimic } & F: CAGUGCAAUGUUAAAAGGGCAU \\
\hline & R: AUAGCCCUGUACAAUGCUGCUUU \\
\hline \multirow[t]{2}{*}{ miR-130a mimic NC } & F: UUCUCCGAACGUGUCACGUTT \\
\hline & R: ACUUUGACAAUACUAUAGUGGAA \\
\hline \multirow[t]{2}{*}{ ESR1-3'UTR-WT } & F: TAAACGCGTGTAACGTGAATACCAC \\
\hline & R: TCGATGCGTAACGATGTTCCTCAGTG \\
\hline \multirow[t]{2}{*}{ ESR1-3'UTR-MT } & F: TATTACCGATACGGAAAAGCAATGT \\
\hline & R: TGAAGCAACGGAAATGCATAGA \\
\hline \multirow[t]{2}{*}{ AR-3'UTR-WT } & F: TACGAAAAACTTGAATGACAATAC \\
\hline & R: GAAAATGAACTTGAGACAAATG \\
\hline \multirow[t]{2}{*}{ AR-3'UTR-MT } & F: GCGATGACACTGCTCCTATAGCGAAT \\
\hline & R: TGGGATCCCACTGCTCCCATGGCTTA \\
\hline
\end{tabular}

miR, microRNA; NC, negative control; UTR, untranslated region; WT, wild-type; MT, mutant; F, forward; R, reverse.

confluence, transfected cells were serum-starved for $24 \mathrm{~h}$ at $5 \% \mathrm{CO}_{2}, 37^{\circ} \mathrm{C}$ and then cultured in 96-well plates $\left(1 \times 10^{3}\right.$ cells/well). Subsequently, at selected time points (24, 48, 72, 96 and $120 \mathrm{~h}), 20 \mu \mathrm{l} \mathrm{CCK}-8$ reagent (Dojindo Molecular Technologies, Inc.) were added in each well, and incubated at $37^{\circ} \mathrm{C}$ for $2 \mathrm{~h}$ to measure the rate of cell proliferation. Absorbance was measured at a wavelength of $490 \mathrm{~nm}$ using a SpectraMax 190 microplate reader (Bio-Rad Laboratories, Inc.). For the cell clonogenic survival assays, transfected cells were seeded $\left(2 \times 10^{3}\right.$ cells/plate) into 6 -well plates. Following culture for 2 weeks at $5 \% \mathrm{CO}_{2}, 37^{\circ} \mathrm{C}$, cell colonies (-50 cells are visible from one cell) were fixed in $2 \%$ formaldehyde in PBS for $2 \mathrm{~min}$ and the stained with $0.5 \%$ crystal violet for $30 \mathrm{~min}$ (both at room temperature). Colonies were visually counted.

Transwell invasion assay. Cells were plated ( $2 \times 10^{5}$ cells/well) in serum-free medium in Transwell chambers $(8-\mu \mathrm{m}$ pore size; BD Biosciences) pre-coated with Matrigel $\left(4^{\circ} \mathrm{C}\right.$ for $2 \mathrm{~h}$ ). Complete DMEM/F12 medium containing 10\% FBS was added to 24-well plates as a chemoattractant (lower chamber). After $24 \mathrm{~h}$ of incubation at $5 \% \mathrm{CO}_{2}, 37^{\circ} \mathrm{C}$, the cells were fixed with $4 \%$ paraformaldehyde for $1 \mathrm{~h}$ at room temperature. The cells on the apical side of each insert were removed by mechanical scraping. The cells that migrated to the basal side of the membrane were stained with $0.1 \%$ crystal violet at room temperature and visualized under a Leica DMI 3000B light microscope (Leica Microsystems, Inc.; magnification, x200).

Luciferase assays. By searching for potential miRNAs, the miR-130a-3p targeting site was identified within the 3'untranslated region (UTR) of ESR1 and AR, as detected by three software algorithms (TargetScan v3.1, targetscan.org; Pictar, pictar.mdc-berlin.de; and MiRanda, miranda.org). To examine whether miR-130a-3p could modulate ER $\alpha$ and AR expression, a DNA fragment comprising a partial wild-type (WT) 3'UTR of ESR1or AR was constructed, as well as a corresponding mutant (MT) 3'UTR of ESR1 or AR. The plasmids were synthesized and cloned into the pGL3-REPORT luciferase vector (Promega Corporation) containing the luciferase gene to generate pGL3-ESR1/AR-3'UTR-WT and pGL3-ESR1/AR-3'UTR-MT. 293T cells (5x104/well) were seeded into 24-well plates and transfected with $0.2 \mu \mathrm{g}$ of either pGL3-ESR1/AR-3'UTR-WT or pGL3-ESR1/AR-3'UTR-MT. The luciferase reporter assay was performed as previously described $(16,21,22)$

Xenograft tumor growth assays. The animal experiments were performed in strict accordance with the Guideline for the Care and Use of Laboratory Animals of China. The protocol was approved by the Committee on the Ethics of Animal Experiments of Shanghai Jiaotong University [approval no. SCXK (hu) 2018-0007]. All efforts were made to minimize animal suffering. A total of 10 female BALB/c nude mice (age, 5 weeks; weight, $15 \mathrm{~g}$ ) were obtained from the Chinese Academy of Sciences. Animals were maintained at standard controlled conditions (room temperature, $22 \pm 1^{\circ} \mathrm{C}$; relative humidity, 50-60\%) on a $12 \mathrm{~h}$ light/dark cycle and free access to food and water. HeLa cells were harvested and resuspended $\left(5 \times 10^{6}\right.$ cells $\left./ 200 \mu \mathrm{l}\right)$ in sterile saline. Mice $(\mathrm{n}=5$ per group; miR-130a-targeting antagomiR group and the 
A



$\mathrm{C}$

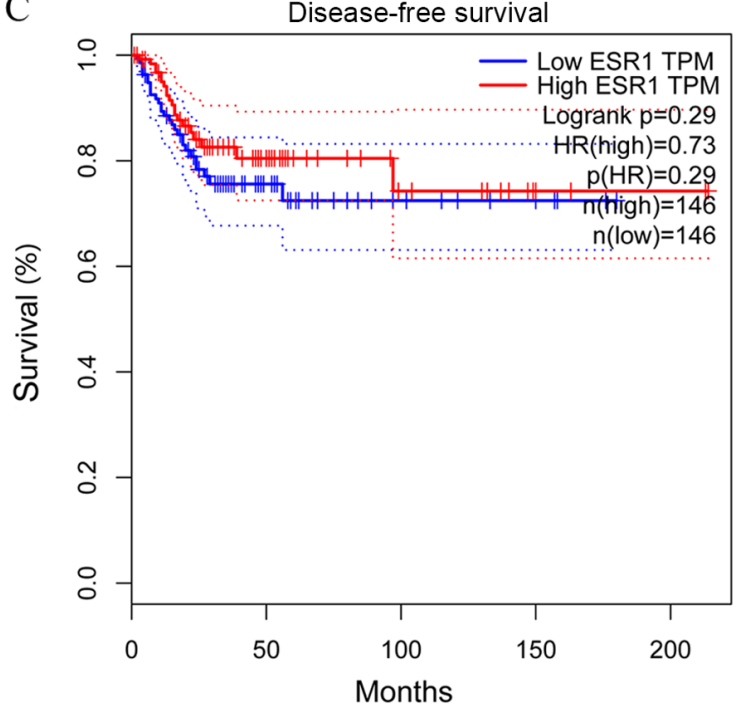

B



$\mathrm{D}$

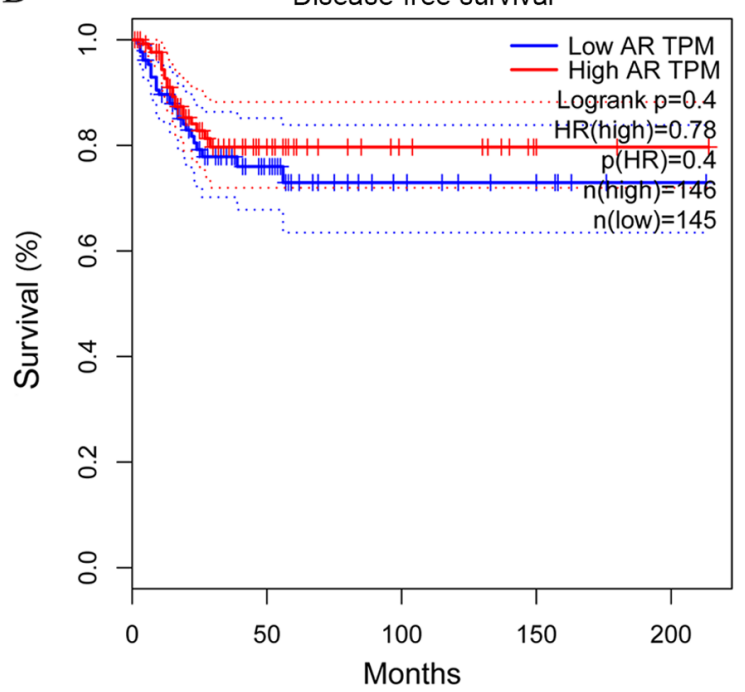

Figure 1. In silico analysis of ESR1 and AR expression, and the association with patient survival in CC. Genomics of cross-cancer alteration summary of (A) ESR1 and (B) AR genes in tumor and healthy tissues. (C) DFS of the high-ESR1 (HR, 0.73; 95\% CI, 0.61-0.90) and low-ESR1 (95\% CI, 0.62-0.82) groups. (D) DFS of the high-AR (HR, 0.78; 95\% CI, 0.72 to 0.88) and low-AR (95\% CI, 0.63-0.83) groups. ESR1, estrogen receptor $\alpha$ gene; AR, androgen receptor; DFS, disease-free survival; CI, confidence interval; HR, hazard ratio.

control antagomiR group) were subcutaneously injected with $200 \mu \mathrm{l} \mathrm{HeLa}$ cells in the subdermal space on the medial side of the neck. After $\sim 1$ week, when tumors reached an average volume of $\sim 20 \mathrm{~mm}^{3}$, each tumor was directly injected with antagomiR-130a (cat. no. B05001) or control antagomiR (cat. no. B04007) (both from Cytiva; $40 \mathrm{ml}$ PBS containing $1 \mu \mathrm{g}$ antagomiR-130a or control antagomiR) on day 0 (when the tumors reached an average volume of $20 \mathrm{~mm}^{3}$ ), 5 and 9 (23). Tumor volume was measured every 7 days until the end of the experiment and was calculated using the following formula: Volume $=\left(\right.$ largest diameter $\times$ smallest diameter $\left.{ }^{2}\right) \times 0.5$. At the end of the xenograft experiment (day 21), mice were sacrificed by $\mathrm{CO}_{2}$ inhalation ( $30 \%$ volume displacement per minute). Following sacrifice, tumor weight was determined.

In silico analysis of ESR $1, A R$ and disease-free survival (DFS) of patients with CC. In silico analysis of the association between ESR1 or AR and DFS of patients with cervical cancer was performed using online published data obtained from Gene Expression Profiling Interactive Analysis (GEPIA; gepia.cancer-pku.cn) and The Cancer Genome Atlas (TCGA) network (cBioPortal for Cancer Genomics; cbioportal. org) (24-26). Overall survival (OS), DFS, disease-specific survival (DSS) and progression-free survival (PFS) were analyzed using the cBioPortal for Cancer Genomics database $(25,26)$.
Statistical analysis. Comparisons between two groups were analyzed using an unpaired Student's t-test. Comparisons among multiple groups were analyzed using one-way ANOVA followed by Tukey's post hoc test. DFS was assessed by using the Kaplan-Meier method with the log-rank test Data are presented as the mean \pm standard deviation. $\mathrm{P}<0.05$ was considered to indicate a statistically significant difference. All statistical analyses were performed using SPSS software (version 16.0; SPSS, Inc.). All experiments were carried out in triplicate and repeated at least three times.

\section{Results}

In silico analysis of ESRI and AR expression, and association with survival of patients with $C C$. To establish whether steroid hormone receptors affected CC progression, the GEPIA database was used to examine cross-cancer alteration summaries of ESR1 and AR. Patients with CC displayed notably lower ESR1 and AR expression levels compared with healthy individuals (Fig. 1A and B). The associations between ESR1 and AR expression and DFS of patients with CC $(n=292)$ were assessed in an independent dataset obtained from the GEPIA database (24). The DFS of the high-ESR1 group [hazard ratio (HR), 0.73; 95\% confidence interval (CI), 0.61-0.90] and the low-ESR1 group (95\% CI, 0.62-0.82) are presented in Fig. 1C. The DFS of the high-AR group (HR, 0.78; 95\% CI, 0.72-0.88) 



CIN I

CIN II

CIN III

CC


C


Figure 2. ER $\alpha$ and AR are significantly decreased in CC. Immunohistochemical analysis of (A) ER $\alpha$ and (B) AR in healthy cervical, CIN and squamous cell cervical carcinoma tissues (magnification, x200). (C) Quantification of immunohistochemical staining in healthy cervical epithelium ( $\mathrm{n}=20$ ), CIN I ( $=20$ ), CIN II ( $n=20)$, CIN III ( $n=30)$ and CC $(n=60)$ tissues. ${ }^{*} \mathrm{P}<0.05$ and ${ }^{* *} \mathrm{P}<0.01$. ER $\alpha$, estrogen receptor $\alpha$; AR, androgen receptor; CC, cervical cancer; CIN, cervical intraepithelial neoplasia; IHC, immunohistochemistry; CI, confidence interval.

and the low-AR group (95\% CI, 0.63-0.83) are presented in Fig. 1D. In another TCGA network (cBioPortal for Cancer Genomics) $(25,26)$, the OS, DFS, DSS and PFS of patients with CC from the cBioPortal database were also analyzed (data not shown). Although not significant, patients with CC and lower ESR1 and AR expression displayed poorer survival rates compared with patients with $\mathrm{CC}$ and higher ESR1 and AR expression.

$E R \alpha$ and $A R$ expression is significantly decreased in $C C$. The protein expression levels of ER $\alpha$ and AR in CC were analyzed via IHC. ER $\alpha$ - and AR-positive immunostaining was observed in the cell nucleus (Fig. 2A and B). The protein expression levels of $\mathrm{ER} \alpha$ and $\mathrm{AR}$ were decreased in a sequential manner from healthy cervical to CIN and further to CC tissues (Fig. 2C), suggesting that low ER $\alpha$ and AR expression was associated with high-grade lesions.

$E R \alpha$ and $A R$ are targets of $m i R-130 a-3 p$. miRNAs can regulate gene expression by inhibiting translation or inducing mRNA degradation at the post-transcriptional level (27). The miR-130a-3p targeting site was identified within the 3'UTRs of ESR1 and AR (Fig. 3A). 293T cells were co-transfected with WT or MT vector and miR-130am or miR-130am NC. miR-130am significantly decreased the relative luciferase activity of ESR1-3'UTR-WT and AR-3'UTR-WT compared with miR-130am NC. By contrast, miR-130am did not significantly alter the luciferase activity of ESR1-3'UTR-MT or AR-3'UTR-MT compared with miR-130am NC (Fig. 3B and C).

To further investigate the functional role of deregulated miR-130a-3p in CC cells, the effects of miR-130ai on the expression of ER $\alpha$ and AR were examined. miR-130ai notably increased the protein expression levels of ER $\alpha$ and AR compared with miR-130ai NC in HeLa and SiHa cells (Fig. 3D), supporting its role as a functional suppressor of $\mathrm{ER} \alpha$ and AR. Additionally, the levels of miR-130a-3p in 20 healthy cervical tissues and $30 \mathrm{CC}$ tissues were detected via RT-qPCR. The results indicated that miR-130a-3p expression levels were significantly higher in $\mathrm{CC}$ tissues compared with healthy cervical tissues (Fig. 3E).

miR-130a-3p knockdown inhibits CC cell proliferation and invasion. To identify the biological function of miR-130a-3p, HeLa and SiHa cells were transfected with miR-130ai. Compared with miR-130ai NC, miR-130ai significantly inhibited HeLa and $\mathrm{SiHa}$ cell proliferation, as indicated by the CCK-8 and clonogenic assay results (Fig. 4A and B). Subsequently, the role of miR-130a-3p in the HeLa and $\mathrm{SiHa}$ cell invasion was investigated following transfection with miR-130ai. miR-130ai significantly decreased cell invasion compared with miR-130ai NC (Fig. 4C), suggesting that miR-130a-3p enhanced cell invasion. 
A



$\mathrm{B}$



$\mathrm{D}$

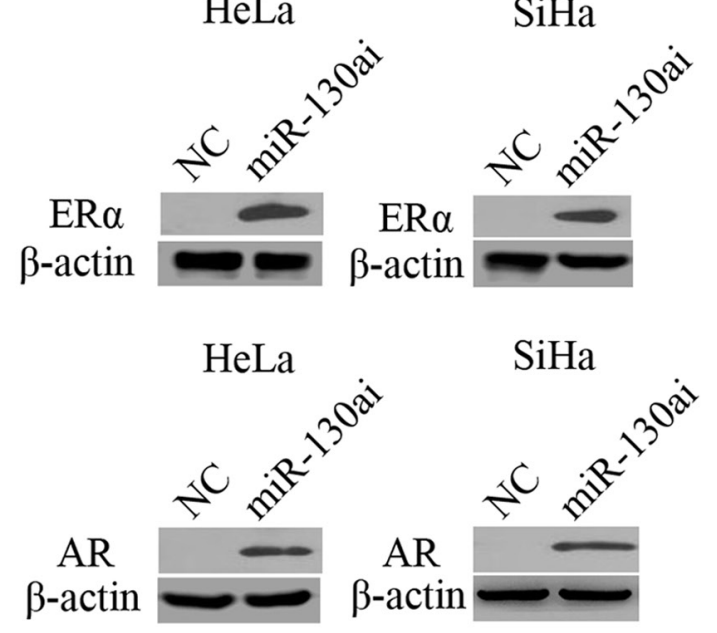

$\mathrm{C}$



E

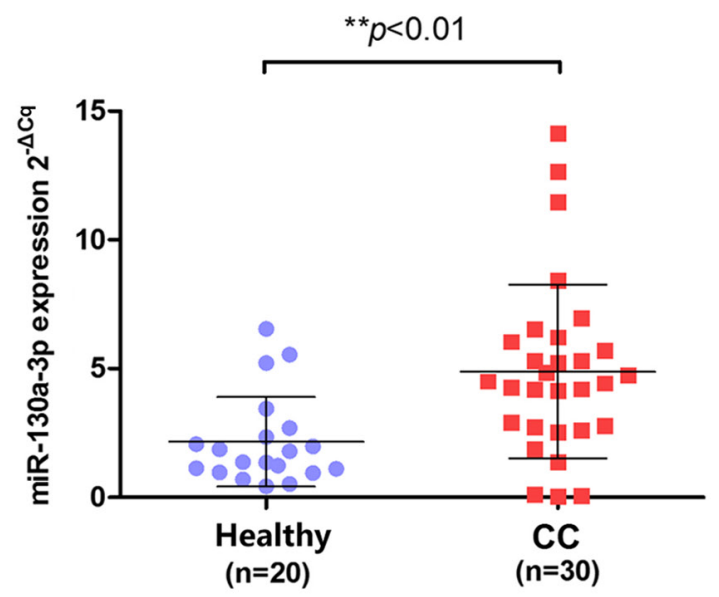

Figure 3. ER $\alpha$ and AR are targets of miR-130a-3p. (A) Putative miR-130a-3p binding site in the 3'UTRSs of ESR1 and AR, as predicted by TargetScan, Pictar and MiRanda. Luciferase assay of 293T cells co-transfected with (B) pGL3-ESR1-3'UTR-WT, pGL3-ESR1-3'UTR-MT, (C) pGL3-AR-3'UTR-WT or pGL3-AR-3'UTR-MT and miR-130am or NC. (D) ER $\alpha$ and AR protein expression levels in HeLa and SiHa cells following transfected with miR-130ai NC or miR-130ai. (E) miR-130a-3p expression levels in $\mathrm{CC}(\mathrm{n}=30)$ and healthy cervical $(\mathrm{n}=20)$ tissues. ${ }^{* *} \mathrm{P}<0.01 \mathrm{vs.} \mathrm{NC}$. EER $\alpha$, estrogen receptor $\alpha$; AR, androgen receptor; miR, microRNA; 3'UTR, 3'untranslated region; WT, wild-type; MT, mutant; miR-130am, miR-130a mimics; miR-130ai, miR-130a inhibitor; NC, negative control; CC, cervical cancer.

ER $\alpha$ and $A R$ are functionally targets of miR-130a-3p, and are involved in CC cell proliferation and invasion. To investigate the biological function of ER $\alpha$ and $A R$, vector transduction was performed to overexpress ER $\alpha$ and AR in HeLa and SiHa cells. Compared with the NC group, ER $\alpha$ overexpression and AR overexpression significantly inhibited $\mathrm{HeLa}$ and $\mathrm{SiHa}$ cell proliferation and invasion, as assessed by performing CCK-8 and Transwell assays (Fig. 5).

To address whether the biological function effects of ER $\alpha$ and AR expression were predominately due to the regulation of miR-130a-3p, the present study investigated whether miR-130a-3p, ER $\alpha$ and AR functioned via the same signaling pathway to modulate $\mathrm{CC}$ cell proliferation and invasion. The results suggested that miR-130am significantly rescued CC cell proliferation and invasion in ER $\alpha$ - and AR-overexpression HeLa and SiHa cells (Fig. 5). The results suggested that ER $\alpha$ and AR were functional targets of miR-130a-3p in CC cells.

Blocking miR-130a-3p inhibits tumorigenicity in vivo. Animal studies were conducted to assess the effect of miR-130a-3p on tumor growth in nude mice. HeLa cells were subcutaneously injected into subdermal space on the medial side of the neck of nude mice to establish tumors, which were treated by direct intratumoral injection when they became clearly 
A

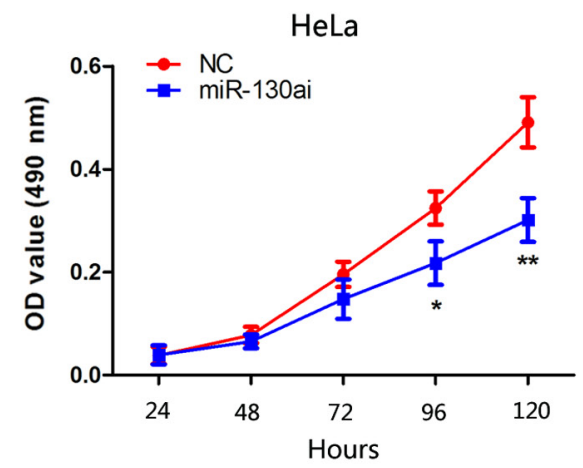

$\mathrm{SiHa}$



B



$\mathrm{NC}$

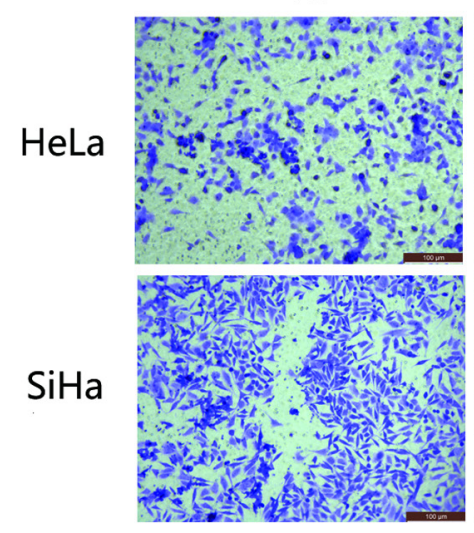



$\mathrm{SiHa}$



$\mathrm{C}$

Figure 4. miR-130a-3p promotes cervical cancer cell proliferation and invasion. HeLa and SiHa cell proliferation, as determined by performing (A) Cell Counting Kit-8 and (B) clonogenic assays (6-wells). (C) HeLa and SiHa cell invasion (magnification, $\mathrm{x} 200$ ). ${ }^{*} \mathrm{P}<0.05$ and ${ }^{* *} \mathrm{P}<0.01$. miR, microRNA; OD, optical density; NC, negative control; HPF, high-power fields; miR-130ai, miR-130a inhibitor.

palpable. After monitoring tumor growth for 21 days, the antagomiR-130a group displayed significantly decreased tumor size and weight compared with the control antagomiR group (Fig. 6).

\section{Discussion}

To the best of our knowledge, the present study suggested for the first time that miR-130a-3p promoted CC cell proliferation and invasion by directly targeting ER $\alpha$ and AR. Despite advances in diagnostic and screening techniques, and the availability of vaccines, $\mathrm{CC}$ remains the fourth largest cause of cancer-related deaths in women worldwide (1). Numerous established risk factors of $\mathrm{CC}$ have been reported, including exposure to HPV, a high number of sexual partners and young age at onset of sexual activity (2). Risk factors that are associated with progression from HPV infection to pre-cancer include oral contraceptive use and smoking (28). The importance of estrogen and ER $\alpha$ at all carcinogenic steps was strongly supported in certain mouse models $(29,30)$; however, few human studies have been conducted. Therefore, the exact role of hormonal factors in progression to pre-cancer and cancer is not completely understood.

$\mathrm{ER} \alpha$ is usually expressed in healthy cervical tissues, but its expression is decreased or absent in invasive $\mathrm{CC}$, which indicated that ER $\alpha$ expression is lost during the development of CC (31). Zhai et al (32) reported that restoration of ESR1 expression in ER $\alpha$-negative CC reduced cell invasiveness in cell culture, and concluded that loss of ER $\alpha$ expression serves a major role in mediating CC invasion and progression. However, other studies have indicated that estrogenic stimulation can influence cervical tumorigenesis $(32,33)$ in agreement with previous studies, the present study suggested that the expression of $E R \alpha$ and $A R$ was decreased in a sequential manner 




$\mathrm{C} \quad \mathrm{NC}$


B



AR



Figure 5. ER $\alpha$ and AR are functional targets of miR-130a-3p, and are involved in cervical cancer cell proliferation and invasion. (A) HeLa and (B) SiHa cel proliferation. (C) HeLa and SiHa cell invasion (magnification, x200). ${ }^{*} \mathrm{P}<0.05$ and ${ }^{* *} \mathrm{P}<0.01$ vs. NC. ER $\alpha$, estrogen receptor $\alpha$; AR, androgen receptor; miR, microRNA; OD, optical density; NC, negative control; miR-130am, miR-130a mimics.




Figure 6. Blocking miR-130a-3p inhibits tumorigenicity in a mouse xenograft model. Tumor cells were injected subcutaneously into mice in the subdermal space on the medial side of the neck. The short and long diameters of the tumors were measured every 7 days, and the tumor volume $\left(\mathrm{cm}^{3}\right)$ were calculated. (A) Representative image of tumors. Tumor (B) volume and (C) weight. " $\mathrm{P}<0.05$ vs. NC. miR, microRNA; NC, negative control. 
from healthy cervical tissues to CIN tissues and further to $\mathrm{CC}$ tissues, suggesting that the loss of ER $\alpha$ and AR served a major role in mediating $\mathrm{CC}$ progression. The role of $\mathrm{AR}$ in $\mathrm{CC}$ is not completely understood. A previous study reported that long-term androgen treatment of female-to-male transsexuals is associated with morphological alterations of the cervix (34). In another study, Noël et al (10) demonstrated that the loss of AR expression is a frequent and common event in high-grade squamous intraepithelial lesion and invasive squamous CC, resulting from complex interactions between high risk HPVs and AR. However, the underlying regulatory mechanisms and the downstream biological effects of the loss of ER $\alpha$ and AR in CC malignancy are not completely understood.

Aberrant expression of miR-130a has been reported in several types of cancer, including gastric (35), esophageal (36) and breast cancer $(21)$, as well as in CC $(15,37)$. However, the role of miR-130a in tumor development and progression is contradictory, as it can serve as an oncogene or tumor suppressor gene by regulating various canonical signaling pathways or target genes $(38,39)$. miR-130a is a potential oncomiR candidate in adriamycin-resistant breast cancer and platinum-resistant ovarian cancer, whereas it serves as a suppressive miRNA in cisplatin-resistant hepatoma cells (39). To date, the role of miR-130a in the progression of $\mathrm{CC}$ carcinogenesis is not completely understood. Yin et al (40) reported that high expression of miR-130a was significantly associated with lymph node metastasis and an advanced clinical stage of CC. The results of the present study indicated that the expression of miR-130a in $\mathrm{CC}$ tissues was obviously higher compared with healthy cervical tissues, and miR-130a knockdown inhibited CC cell proliferation and invasion in vitro and in vivo compared with miR-130ai $\mathrm{NC}$ and control antagomiR, respectively. Moreover, the present study indicated that miR-130a could directly bind to the 3'UTR of ESR1 and AR mRNA, suggesting that miR-130a mediated $\mathrm{CC}$ progression by functionally regulating $\mathrm{ER} \alpha$ and $\mathrm{AR}$.

In conclusion, the present study indicated that miR-130a-3p may contribute to tumor progression by suppressing $\mathrm{ER} \alpha$ and $\mathrm{AR}$, and it may serve as a promising candidate target for the treatment of patients with CC.

\section{Acknowledgements}

Not applicable.

\section{Funding}

The present study was supported by the National Natural Science Foundation of China (grant nos. 81172477, 81572547 and 81402135), the Project of Shanghai Key Clinical Department (grant no. shslczdzk06302), the Project of the Science and Technology Commission of Shanghai Municipality (grant nos. 11ZR1440800 and 13JC1401303), the Project of Outstanding Subject Leaders of the Shanghai Health System (grant no. XBR2013097) and the Shanghai Jiao Tong University Medicine-Engineering Fund (grant no. YG2017MS41).

\section{Availability of data and materials}

The datasets used and/or analyzed during the current study are available from the corresponding author on reasonable request.

\section{Authors' contributions}

QF and YW conceived and designed the study. QF, TH, XS, XY, JW, YaL, TN and SG performed the experiments. QF, TH, XS and YuL analyzed the data. QF, TH and YW wrote the manuscript. All authors read and approved the final manuscript.

\section{Ethics approval and consent to participate}

The present study was approved (approval no. GKLW 2017-125) by the Human Investigation Ethical Committee of the International Peace Maternity \& Child Hospital Affiliated to Shanghai Jiao Tong University School of Medicine (Shanghai, China). The animal experiment protocol was approved by the Committee on the Ethics of Animal Experiments of Shanghai Jiaotong University [approval no. SCXK (hu) 2018-0007].

\section{Patient consent for publication}

Not applicable.

\section{Competing interests}

The authors declare that they have no competing interests.

\section{References}

1. Siegel RL, Miller KD and Jemal A: Cancer statistics, 2019. CA Cancer J Clin 69: 7-34, 2019.

2. Zhao F and Qiao Y: Cervical cancer prevention in China: A key to cancer control. Lancet 393: 969-970, 2019.

3. Brasseur K, Gevry N and Asselin E: Chemoresistance and targeted therapies in ovarian and endometrial cancers. Oncotarget 8: 4008-4042, 2017.

4. Wu MH, Huang CJ, Liu ST, Liu PY, Ho CL and Huang SM: Physical and functional interactions of human papillomavirus E2 protein with nuclear receptor coactivators. Biochem Biophys Res Commun 356: 523-528, 2007.

5. Remoue F, Jacobs N, Miot V, Boniver J and Delvenne P: High intraepithelial expression of estrogen and progesterone receptors in the transformation zone of the uterine cervix. Am J Obstet Gynecol 189: 1660-1665, 2003.

6. Liang J and Shang Y: Estrogen and cancer. Annual Rev Physiol 75: 225-240, 2013.

7. Rosenfeld MG and Glass CK: Coregulator codes of transcriptional regulation by nuclear receptors. J Biol Chem 276: 36865-36868, 2001

8. Yang XM, Wu ZM, Huang H, Chu XY, Lou J, Xu LX, Chen YT, Wang LQ and Huang OP: Estrogen receptor 1 mutations in 260 cervical cancer samples from Chinese patients. Oncol Lett 18: 2771-2776, 2019.

9. Sood S, Patel FD, Ghosh S, Arora A, Dhaliwal LK and Srinivasan R: Epigenetic alteration by DNA methylation of ESR1, MYOD1 and hTERT Gene promoters is useful for prediction of response in patients of locally advanced invasive cervical carcinoma treated by chemoradiation. Clin Oncol (R Coll Radiol) 27: 720-727, 2015.

10. Noël JC, Bucella D, Fayt I, Simonart T, Buxant F, Anaf V and Simon P: Androgen receptor expression in cervical intraepithelial neoplasia and invasive squamous cell carcinoma of the cervix. Int J Gynecol Pathol 27: 437-441, 2008.

11. Bartel DP: MicroRNAs: Genomics, biogenesis, mechanism, and function. Cell 116: 281-297, 2004.

12. Lu J, Getz G, Miska EA, Alvarez-Saavedra E, Lamb J, Peck D, Sweet-Cordero A, Ebert BL, Mak RH, Ferrando AA, et al: MicroRNA expression profiles classify human cancers. Nature 435: 834-838, 2005.

13. Bao W, Wang HH, Tian FJ, He XY, Qiu MT, Wang JY, Zhang HJ, Wang LH and Wan XP: A TrkB-STAT3-miR-204-5p regulatory circuitry controls proliferation and invasion of endometrial carcinoma cells. Mol Cancer 12: 155, 2013. 
14. Lv T, Song K, Zhang L, Li W, Chen Y, Diao Y, Yao Q and Liu P: miRNA-34a decreases ovarian cancer cell proliferation and chemoresistance by targeting HDAC1. Biochem Cell Biol 96: 663-671, 2018

15. He L, Wang HY, Zhang L, Huang L, Li JD, Xiong Y, Zhang MY, Jia WH, Yun JP, Luo RZ and Zheng M: Prognostic significance of low DICER expression regulated by miR-130a in cervical cancer. Cell Death Dis 5: e1205, 2014.

16. Bao W, Zhang Y, Li S, Fan Q, Qiu M, Wang Y, Li Y, Ji X, Yang Y, Sang Z, et al: miR1075p promotes tumor proliferation and invasion by targeting estrogen receptoralpha in endometrial carcinoma. Oncol Rep 41: 1575-1585, 2019.

17. Wright JD, Matsuo K, Huang Y, Tergas AI, Hou JY, Khoury-Collado F, St Clair CM, Ananth CV, Neugut AI and Hershman DL: Prognostic performance of the 2018 international federation of gynecology and obstetrics cervical cancer staging guidelines. Obstet Gynecol 134: 49-57, 2019.

18. Fan Q, Qiu MT,Zhu Z,Zhou JH, Chen L, Zhou Y, Gu W, Wang LH, $\mathrm{Li} \mathrm{ZN}, \mathrm{Xu} \mathrm{Y}$, et al: Twist induces epithelial-mesenchymal transition in cervical carcinogenesis by regulating the TGF- $\beta / \mathrm{Smad} 3$ signaling pathway. Oncol Rep 34: 1787-1794, 2015.

19. Bao W, Qiu H, Yang T, Luo X, Zhang H and Wan X: Upregulation of TrkB promotes epithelial-mesenchymal transition and anoikis resistance in endometrial carcinoma. PLoS One 8: e70616, 2013.

20. Livak KJ and Schmittgen TD: Analysis of relative gene expression data using real-time quantitative PCR and the 2(-Delta Delta C(T)) method. Methods 25: 402-408, 2001.

21. Pan Y, Wang R, Zhang F, Chen Y, Lv Q, Long G and Yang K: MicroRNA-130a inhibits cell proliferation, invasion and migration in human breast cancer by targeting the RAB5A. Int J Clin Exp Pathol 8: 384-393, 2015.

22. Zheng L, Kang Y, Zhang L and Zou W: MiR-133a-5p inhibits androgen receptor (AR)-induced proliferation in prostate cancer cells via targeting FUsed in Sarcoma (FUS) and AR. Cancer Biol Ther 21: 34-42, 2020.

23. Mercatelli N, Coppola V, Bonci D, Miele F, Costantini A, Guadagnoli M,Bonanno E, Muto G, Frajese GV, De Maria R, et al: The inhibition of the highly expressed miR-221 and miR-222 impairs the growth of prostate carcinoma xenografts in mice. PLoS One 3: e4029, 2008.

24. Tang Z, Li C, Kang B, Gao G, Li C and Zhang Z: GEPIA: A web server for cancer and normal gene expression profiling and interactive analyses. Nucleic Acids Res 45: W98-W102, 2017.

25. Cerami E, Gao J, Dogrusoz U, Gross BE, Sumer SO, Aksoy BA, Jacobsen A, Byrne CJ, Heuer ML, Larsson E, et al: The cBio cancer genomics portal: An open platform for exploring multidimensional cancer genomics data. Cancer Discov 2: 401-404, 2012

26. Gao J, Aksoy BA, Dogrusoz U, Dresdner G, Gross B, Sumer SO, Sun Y, Jacobsen A, Sinha R, Larsson E, et al: Integrative analysis of complex cancer genomics and clinical profiles using the cBioPortal. Sci Signal 6: pl1, 2013.

27. Laengsri V, Kerdpin U, Plabplueng C, Treeratanapiboon L and Nuchnoi P: Cervical cancer markers: Epigenetics and microRNAs. Lab Med 49: 97-111, 2018.
28. Luhn P, Walker J, Schiffman M, Zuna RE, Dunn ST, Gold MA, Smith K, Mathews C, Allen RA, Zhang R, et al: The role of co-factors in the progression from human papillomavirus infection to cervical cancer. Gynecol Oncol 128: 265-270, 2013.

29. Chung SH, Wiedmeyer K, Shai A, Korach KS and Lambert PF: Requirement for estrogen receptor alpha in a mouse model for human papillomavirus-associated cervical cancer. Cancer Res 68: 9928-9934, 2008.

30. Chung SH, Franceschi S and Lambert PF: Estrogen and ERalpha: Culprits in cervical cancer? Trends Endocrinol Metab 21: 504-511, 2010.

31. Lopez-Romero R, Garrido-Guerrero E, Rangel-Lopez A, Manuel-Apolinar L, Piña-Sánchez P, Lazos-Ochoa M Mantilla-Morales A, Bandala C and Salcedo M: The cervical malignant cells display a down regulation of ER- $\alpha$ but retain the ER- $\beta$ expression. Int J Clin Exp Pathol 6: 1594-1602, 2013.

32. Zhai Y, Bommer GT, Feng Y, Wiese AB, Fearon ER and Cho KR: Loss of estrogen receptor 1 enhances cervical cancer invasion. The American journal of pathology 177: 884-895, 2010.

33. Deligeoroglou E, Michailidis E and Creatsas G: Oral contraceptives and reproductive system cancer. Annals of the New York Academy of Sciences 997: 199-208, 2003.

34. Miller N, Bedard YC, Cooter NB and Shaul DL: Histological changes in the genital tract in transsexual women following androgen therapy. Histopathology 10: 661-669, 1986.

35. Jiang H, Yu WW, Wang LL and Peng Y: miR-130a acts as a potential diagnostic biomarker and promotes gastric cancer migration, invasion and proliferation by targeting RUNX3. Oncology reports 34: 1153-1161, 2015.

36. Liu SG, Qin XG, Zhao BS, et al.: Differential expression of miRNAs in esophageal cancer tissue. Oncology letters 5: 16391642,2013

37. Chi C, Mao M, Shen Z, Chen Y, Chen J and Hou W: HOXD-AS1 Exerts Oncogenic Functions and Promotes Chemoresistance in Cisplatin-Resistant Cervical Cancer Cells. Human gene therapy 29: $1438-1448,2018$

38. Hofsjo A, Bohm-Starke N, Bergmark K, Masironi B and Sahlin $\mathrm{L}$ : Sex steroid hormone receptor expression in the vaginal wall in cervical cancer survivors after radiotherapy. Acta oncologica 58: 1107-1115, 2019.

39. Zhang HD, Jiang LH, Sun DW, Li J and Ji ZL: The role of miR130a in cancer. Breast cancer 24: 521-527, 2017.

40. Yin S, Zhang Q, Wang Y, Li S and Hu R: MicroRNA-130a regulated by HPV18 E6 promotes proliferation and invasion of cervical cancer cells by targeting TIMP2. Experimental and therapeutic medicine 17: 2837-2846, 2019.

This work is licensed under a Creative Commons Attribution-NonCommercial-NoDerivatives 4.0 International (CC BY-NC-ND 4.0) License. 